\title{
PsoBest - Das Deutsche Psoriasis-Register: Aktuelle Übersicht zur Versorgung
}

\section{The German Psoriasis Registry PsoBest - A Current Overview of Routine Care}

Autoren

Institut

\author{
C. Spehr, S. Knopf, Z. Foos, K. Haack, M. El Hamoumi, J. Hoffmann, S. J. Rustenbach
}

Competenzzentrum Versorgungsforschung in der Dermatologie (CVderm), Institut für Versorgungsforschung in der Dermatologie und bei Pflegeberufen (IVDP), Universitätsklinikum Hamburg-Eppendorf, Hamburg

\section{Bibliografie}

DOI http://dx.doi.org/

10.1055/s-0034-1392776

Akt Dermatol 2015; 41: 330-332

(c) Georg Thieme Verlag KG

Stuttgart · New York

ISSN 0340-2541

\section{Korrespondenzadresse}

\section{Christina Spehr}

Competenzzentrum

Versorgungsforschung in der

Dermatologie (CVderm)

Institut für Versorgungsforschung

in der Dermatologie und bei

Pflegeberufen (IVDP)

Universitätsklinikum

Hamburg-Eppendorf

Martinistraße 52

20246 Hamburg

c.spehr@uke.de

\section{Zusammenfassung \\ $\nabla$}

Bis Sommer 2015 wurden nahezu 4000 Patienten in das Register eingeschlossen, ein Großteil von ihnen durch niedergelassene Dermatologen. Die gemeldeten Therapien umfassten alle verfügbaren Psoriasistherapien in Deutschland.

PsoBest-Patienten repräsentieren Patienten mit hoher Krankheitslast, ihre Behandlung zeigt gute Erfolge und eine hohe Patientenzufriedenheit. Aktuelle Ergebnisse zeigen zudem ein gutes Sicherheitsprofil der systemischen Psoriasistherapie in Deutschland.

Bis Sommer 2015 wurden fast 4000 Patienten in PsoBest registriert. Insgesamt haben sich 681 Dermatologen als kooperierende Registerzentren angemeldet, 263 von ihnen senden regelmäßig Dokumentationen an das CVderm. Auffallend hierbei ist, dass mit 70\% ein Großteil der Dokumentationen von niedergelassenen Dermatologen und deren Teams geleistet wurde. Sie meldeten bereits 2762 Patienten.

Initial erhielten $71 \%$ der Patienten in PsoBest eine konventionelle Systemtherapie ( $\boldsymbol{A}$ Abb. 1). Durch die weitere therapieunabhängige Dokumentation konnten bis Ende 2014 über 7000 Behandlungsjahre beobachtet werden, davon 3000 Patientenjahre unter Biologikatherapie. Patienten, die in PsoBest eine neue Therapie begonnen haben, wiesen eine deutliche Krankheitslast (Biologika/konventionelle Systemtherapie: PASI 15,5/14,1 und BSA 25,5/23,2) und Einschränkung der gesundheitsbezogenen Lebensqualität auf (DLQI 11,6/ 10,8). Patienten unter Biologikatherapie waren dabei häufiger männlich, im Mittel 5 Jahre länger erkrankt und litten häufiger an Psoriasis-Arthritis (PsA). Im Durchschnitt waren sie im Vergleich zu Patienten unter konventioneller Systemtherapie aufgrund der Erkrankung an zusätzlichen $10 \mathrm{Ta}-$ gen im Jahr arbeitsunfähig ( $\bullet$ Tab. 1 ).

Die individuelle Behandlung der Patienten zeigte schnelle und deutliche Erfolge. In den ersten 6 Monaten wurde eine mittlere PASI-Reduktion um $65 \%$ erreicht ( $\bullet$ Abb.2). Auch die Einschränkung der gesundheitsbezogenen Lebensqualität nahm eindeutig ab, im ersten halben Jahr von 12,3 auf 4,5 (DLQI; $0=$ keine bis $32=$ maximale Beeinträchtigung). Diese Behandlungserfolge konnten über die weitere Dokumentationszeit hinaus erhalten werden.

Die deutlichste PASI-Reduktion wurde bereits in den ersten 3 Behandlungsmonaten erreicht. Der mittlere PASI stabilisierte sich nach 6 Monaten unter 5,0. Trotz abnehmenden Stichprobenumfangs reduzierte sich der Fehlerbereich über die Zeit. Dieser Erfolg hielt sich auch noch nach bis zu 7,5 Jahren.

Auch die regelmäßigen Auswertungen zur Pharmakovigilanz zeigen die gute Versorgung. Sowohl Biologikatherapien als auch Therapien mit konventionellen Systemtherapeutika zeigen ein gutes Sicherheitsprofil in der Behandlung von Psoriasis und Psoriasis-Arthritis. Diese Ergebnisse spiegelten sich auch in der Zufriedenheit der Patienten mit ihrer Behandlung wider. Nach dem ersten Halbjahr in PsoBest äußerten sich 4 von 5 Patienten überwiegend positiv über ihre Behandlungssituation $(\bullet$ Abb. 3$)$.

Die in PsoBest eingeschlossenen Patienten repräsentieren Patienten mit starkem Leidensdruck und hohem Schweregrad der Psoriasis. Die Versorgung dieser Patienten durch deutsche Dermatologen zeigte gute Behandlungserfolge und hohe Patientenzufriedenheit. Aktuelle Ergebnisse zeigen zudem ein gutes Sicherheitsprofil der systemischen Psoriasistherapie in Deutschland. 


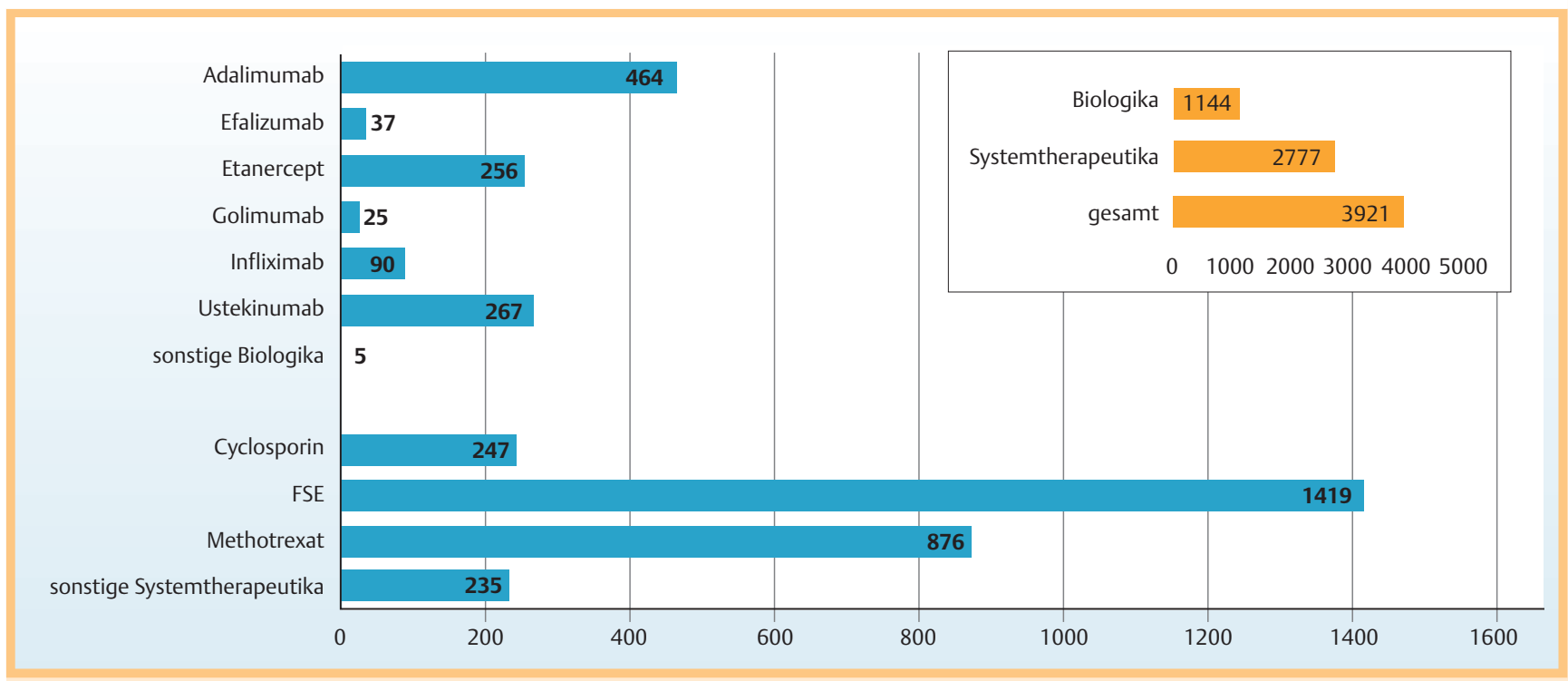

Abb.1 Anzahl der Patienten pro Therapie bei Einschluss in PsoBest (Stand 9.7. 2015).

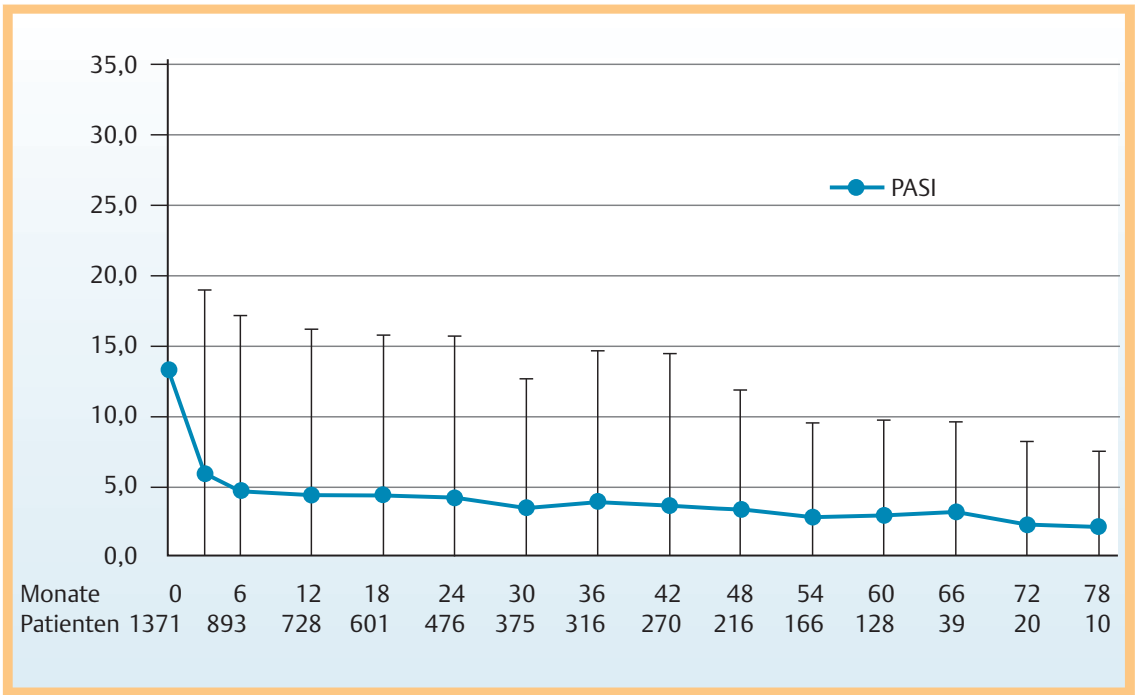

Abb.2 Mittlerer PASI-Verlauf in PsoBest (inkl. 95-\%-Vertrauensintervall).

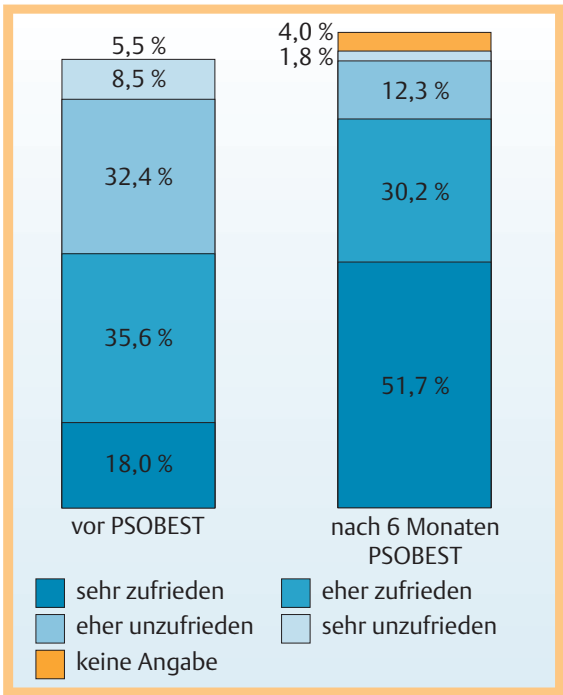

Abb.3 Behandlungszufriedenheit in den letzten 3 Monaten: Innerhalb des ersten Halbjahres steigt der Anteil zufriedener Patienten um 28\%.

\begin{tabular}{|lllllll|} 
& \multicolumn{2}{l}{ Biologika-Einschlüsse } & & \multicolumn{3}{c|}{ Systemtherapie-Einschlüsse } \\
\cline { 2 - 7 } & Männer & Frauen & Gesamt & Männer & Frauen & Gesamt \\
\hline Patienten & 650 & 384 & 1034 & 1528 & 1055 & 2583 \\
\hline Patienten \% & 62,9 & 37,1 & 100,0 & 59,2 & 40,8 & 100.0 \\
\hline Alter (Jahre) & 47,3 & 49,4 & 48,1 & 46,8 & 48,0 & 47,3 \\
\hline Gewicht (kg) & 92,0 & 79,7 & 87,4 & 90,4 & 76,8 & 84,9 \\
\hline Body Mass Index (kg/m²) & 28,7 & 29,1 & 28,9 & 28,3 & 28,0 & 28,2 \\
\hline Adipositas (BMI>30)(\%) & 31,4 & 35,4 & 32,9 & 26,8 & 30,0 & 28,1 \\
\hline AU-Tage im letzten Jahr & 14,1 & 24,3 & 17,2 & 7,1 & 6,9 & 7,0 \\
\hline Erkrankungsdauer (Jahre) & 20,9 & 22,6 & 21,5 & 15,4 & 17,6 & 16,3 \\
\hline Prävalenz PsA (\%) & 33,5 & 44,0 & 37,4 & 12,9 & 16,4 & 14,3 \\
\hline Schmerzen bei PsA (0-10) & 4,6 & 5,2 & 4,9 & 4,5 & 5,5 & 5,0 \\
\hline PASI & 16,5 & 13,8 & 15,5 & 14,7 & 13,4 & 14,1 \\
\hline BSA & 26,7 & 23,4 & 25,5 & 23,8 & 22,3 & 23,2 \\
\hline DLQI & 11,1 & 12,5 & 11,6 & 10,1 & 11,8 & 10,8 \\
\hline DLQI>10 (\%) & 48,6 & 58,3 & 52,2 & 43,3 & 55,0 & 48,0 \\
\hline DLQI>5 (\%) & 24,6 & 17,7 & 22,1 & 26,2 & 22,9 & 24,9 \\
\hline DLQI>1 (\%) & 22,5 & 18,5 & 21,0 & 24,8 & 18,3 & 22,1 \\
\hline
\end{tabular}

Tab. 1 Patienteneigenschaften bei Einschluss in PsoBest (Stand 31.12.2014) Wenn nicht anders vermerkt, sind Mittelwerte dargestellt. 


\section{Interessenkonflikt}

PsoBest wird von folgenden pharmazeutischen Unternehmen finanziell unterstützt: AbbVie, Amgen, Biogen, Celgene, JanssenCilag, medac, Novartis, Pfizer.

\section{Abstract}

\section{The German Psoriasis Registry PsoBest - A Current Overview of Routine Care}

Nearly 4000 patients were included in the German Psoriasis Registry PsoBest until summer 2015, most patients reported by resident physicians. Reported treatments comprised all approved antipsoriatic drugs in Germany.

PsoBest patients represent patients with a high burden of disease, their treatment show soon success and high patient satisfaction. Moreover, current results show the safety of systemic treatment in Germany.

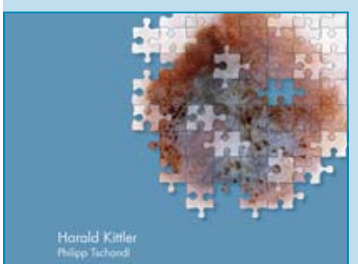

Dermatoskopie - Musteranalyse pigmentierter und unpigmentierter Hautläsionen

Kittler $\mathrm{H}$, Tschandl P

Wien: Facultas, 2015, 2. Aufl., 382 S., 1200 Abb., 64,90€

ISBN 978-3-7089-1142-7

Dermatoskopie

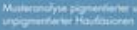

$\underline{5}$

Die Dermatoskopie ist ein wichtiger Bestandteil der Diagnostik von Hauttumoren. Gerade als Anfänger fehlt oft noch ein System. Dieses Buch vermittelt in seinen 11 Kapiteln eine gute Grundlage. Im 1. Kapitel werden allgemeine Grundlagen besprochen, wie z. B. die Untersuchungstechnik, Indikation, Nutzen und die Entwicklung der Methode (Musteranalyse). Es folgt ein Abschnitt über aus dermatologischer Sicht relevante, pigmentierte Hautveränderungen mit Definitionen und Begriffserläuterungen. In der Einheit „Grundlagen der Methode auf Basis der Musteranalyse“ bekommt der Leser gute Hilfsmittel zur Beschreibung des auflichtmikroskopischen Lokalbefundes sowie zur späteren Diagnostik der Hauttumore. Es wird eingegangen auf die Grundmuster wie z. B. Linien, Schollen, Pseudopodien, auf Farben sowie charakteristische Merkmale von nicht-melanozytären Läsionen und melanozytären Läsionen. Darauf folgt eine gängige Erläuterung dermatoskopischer Begriffe. Das 5. und das 6. Kapitel erläutern den algorithmischen Untersuchungs- gang von pigmentierten und unpigmentierten Läsionen. Es folgen wertvolle Tipps und Tricks sowie die Betrachtung spezieller Situationen wie z.B. Läsionen an den Nägeln, akral, im Gesicht sowie entzündliche Läsionen. Die letzten Abschnitte beinhalten digitale Dermatoskopie und Verlaufskontrolle, Fallbeispiele und dermatoskopisch-dermatopathologische Korrelation.

Insgesamt sind an manchen Stellen die Erläuterungen etwas langatmig. Dieses Buch ist nicht geeignet, um einen schnellen Überblick zu bekommen. Das war aber auch gar nicht die Intention der Autoren. Sie legen Wert auf eine fundierte Ausbildung zur Dermatoskopie. Besonders wertvoll sind die Untersuchungsalgorithmen. Sie sind didaktisch gut, um einen Überblick und eine Struktur in die Flut von Informationen zu bekommen. Anhand des Kapitels „Beispiele“ kann man sein frisch erworbenes Wissen überprüfen.

Alles in allem handelt es sich hier um ein sehr empfehlenswertes Buch für alle, die die Auflichtmikroskopie von Grund auf lernen möchten.

Nicole Adler, Wiesbaden 